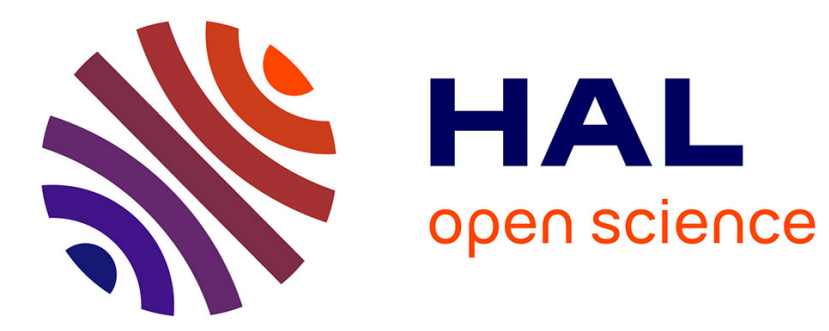

\title{
Shaping the longitudinal electric field component of light
}

Fabian Maucher, Stefan Skupin, Simon Gardiner, Ifan Hughes

\section{To cite this version:}

Fabian Maucher, Stefan Skupin, Simon Gardiner, Ifan Hughes. Shaping the longitudinal electric field component of light. Complex Light and Optical Forces XIII, Feb 2019, San Francisco, United States. pp.1093502, 10.1117/12.2512112 . hal-02083158

\section{HAL Id: hal-02083158 https://hal.science/hal-02083158}

Submitted on 28 Mar 2019

HAL is a multi-disciplinary open access archive for the deposit and dissemination of scientific research documents, whether they are published or not. The documents may come from teaching and research institutions in France or abroad, or from public or private research centers.
L'archive ouverte pluridisciplinaire HAL, est destinée au dépôt et à la diffusion de documents scientifiques de niveau recherche, publiés ou non, émanant des établissements d'enseignement et de recherche français ou étrangers, des laboratoires publics ou privés. 


\title{
Shaping the longitudinal polarization component of light
}

\author{
F. Maucher ${ }^{1, *}$, S. Skupin ${ }^{2}$, S. A. Gardiner ${ }^{3}$, and I. G. Hughes ${ }^{3}$ \\ ${ }^{1}$ Department of Physics and Astronomy, Aarhus University, Ny Munkegade 120, 8000 Aarhus \\ C, Denmark \\ ${ }^{2}$ Univ. Lyon, Université Claude Bernard Lyon 1, CNRS, Institut Lumière Matière, F-69622, \\ Villeurbanne, France \\ ${ }^{3}$ Joint Quantum Centre (JQC) Durham-Newcastle, Department of Physics, Durham \\ University, Durham DH1 3LE, United Kingdom. \\ ${ }^{*}$ Corresponding author: maucher@phys.au.dk
}

\begin{abstract}
This paper illustrates examples of shaping the longitudinal electric field component of light which is relevant for tightly focused beams. Given that the latter is not directly accessible via conventional beam shaping techniques we elaborate on the interplay between the transverse polarization and longitudinal electric field components. A Helmholtz decomposition of the transverse electric field components in the transverse plane permits on the one hand to draw insightful analogies with electro- and magnetostatics and with fluid dynamics. On the other hand, it allows to clearly isolate the remaining degree of freedom in the transverse electric field components for a given longitudinal electric field component and with that to generalize the concepts of radial and azimuthal polarization. We discuss degrees of freedom and show how one can exploit the findings to generate novel customized vector beams. Furthermore, we present a thought experiment to study beams containing evanescent waves.
\end{abstract}

Keywords: Tightly focused light, Laser beam shaping, Optical vortices

\section{INTRODUCTION}

Tightly focused light typically leads to a non-negligible longitudinal polarization component,,$^{1,2}$ where the terms longitudinal and transverse electric field components refer to the components of the electric field that are parallel or perpendicular, respectively, to the direction of the mean Poynting flux. The exception arises for the special case of the beam's transverse electric field components being divergence free in the two-dimensional transverse plane; under these circumstances the longitudinal polarization component exactly vanishes despite tight focusing. ${ }^{3,4}$ The simplest example for that is azimuthal polarization. ${ }^{2}$

Whereas technically this additional electric field component does not actually introduce a new degree of freedom in the sense that all electric and magnetic field components are still fixed if we fix two electric or magnetic field components in a plane, it allows new interesting beams with spatially varying polarization, where all three electric field components are relevant. Classic examples are the so-called needle beam ${ }^{5,6}$ or the Möbius strip in the polarization. ${ }^{7-9}$ Tight focusing of a circularly polarized Gaussian ${ }^{10}$ leads to a nonzero angular momentum through the additional longitudinal field component. Such behaviour is of course of crucial relevance when studying tightly focused beams interacting with matter, and recent experiments have revealed the importance of taking the longitudinal electric field component into account for a complete and accurate picture. ${ }^{11,12}$ It has also been shown that the longitudinal electric field component can even dominate the interaction with matter. ${ }^{13}$ Apart from that, there is current substantial interest in "structured light", $14-16$ that is, generating customized light fields that suit specific needs in applications in a range of fields and extending these ideas to all three electric field components. ${ }^{3,17}$

Usually, the longitudinal polarization $E_{z}$ can be easily computed from the divergence equation as function of the transverse electric field components $\mathbf{E}_{\perp}=\left(E_{x}, E_{y}\right)$ via

$$
E_{z}=E_{z}\left(\mathbf{E}_{\perp}\right) .
$$


In this paper we want to ask the following question: How can we shape $E_{z}$ and what degrees of freedom do we have to control the polarization in the transverse electric field components $\mathbf{E}_{\perp}$ ? In other words, we are asking how we can invert Eq. (1)

$$
\mathbf{E}_{\perp}=\mathbf{E}_{\perp}\left(E_{z}\right) .
$$

That is of course not a well-posed problem, since the solution of Eq. (2) is not unique. However, as we shall see in what follows, the degree of freedom categorizing solutions of Eq. (2) has a simple geometric interpretation. This paper focuses primarily on the electric field. An experimental visualization of the structures presented in this work would entail some atom-light interactions, and it is well known that the electric field dominates the magnetic field when interacting with charges in a medium (see, e.g., ${ }^{18}$ ).

\section{MODEL}

The three electric field components of a tightly focused monochromatic beam (frequency $\omega_{0}$, wavelength $\lambda=$ $\left.2 \pi c / \omega_{0}\right)$ in free space are described by

$$
\begin{aligned}
\nabla^{2} \mathbf{E}\left(\mathbf{r}_{\perp}, z\right)+k_{0}^{2} \mathbf{E}\left(\mathbf{r}_{\perp}, z\right) & =0 \\
\nabla \cdot \mathbf{E}\left(\mathbf{r}_{\perp}, z\right)=\nabla_{\perp} \cdot \mathbf{E}_{\perp}+\partial_{z} E_{z} & =0 .
\end{aligned}
$$

We have introduced $k_{0}^{2}=\omega_{0}^{2} / c^{2}=(2 \pi / \lambda)^{2}$, the transverse coordinates $\mathbf{r}_{\perp}=(x, y)$, and the transverse electric field components $\mathbf{E}_{\perp}=\left(E_{x}, E_{y}\right)$. Note that $\mathbf{E}$ represents the complex amplitude vector of the beam; the full electric field has an additional trivial time-dependence $\exp \left(-i \omega_{0} t\right)$ that is omitted here. For a given amplitude vector in the focal plane* $\mathbf{E}^{\mathrm{f}}\left(\mathbf{r}_{\perp}\right)=\mathbf{E}\left(\mathbf{r}_{\perp}, z=0\right)$, the propagation in the positive $z$ direction can be easily computed in the transverse spatial Fourier domain as

$$
\hat{\mathbf{E}}\left(\mathbf{k}_{\perp}, z\right)=\hat{\mathbf{E}}^{\mathrm{f}}\left(\mathbf{k}_{\perp}\right) \mathrm{e}^{i k_{z}\left(\mathbf{k}_{\perp}\right) z},
$$

where $k_{z}\left(\mathbf{k}_{\perp}\right)=\sqrt{k_{0}^{2}-\mathbf{k}_{\perp}^{2}}$ and $\mathbf{k}_{\perp}=\left(k_{x}, k_{y}\right)$; the symbol ^ denotes the Fourier transform with respect to $\mathbf{r}_{\perp}$.

Before we discuss the equations of motion further, let us first make some general remarks on vector fields. A Helmholtz decomposition permits the decomposition of any three-dimensional vector field $\mathbf{F}$ that is twice continuously differentiable as $\mathbf{F}=-\nabla \phi+\nabla \times \mathbf{A}$. In the two transverse dimensions, the corresponding formula for $\mathbf{F}_{\perp}$ is given by

$$
\mathbf{F}_{\perp}\left(\mathbf{r}_{\perp}\right)=-\nabla_{\perp} V\left(\mathbf{r}_{\perp}\right)+\left(\begin{array}{cc}
0 & 1 \\
-1 & 0
\end{array}\right) \nabla_{\perp} W\left(\mathbf{r}_{\perp}\right)
$$

or, written component-wise

$$
\mathbf{F}_{\perp, i}\left(\mathbf{r}_{\perp}\right)=-\partial_{i} V\left(\mathbf{r}_{\perp}\right)+\sum_{j} \epsilon_{i j} \partial_{j} W\left(\mathbf{r}_{\perp}\right) .
$$

Here, $V\left(\mathbf{r}_{\perp}\right)$ and $W\left(\mathbf{r}_{\perp}\right)$ denote arbitrary (sufficiently well behaved) scalar potentials and $\epsilon_{i j}$ represents the usual Levi-Civita symbol. We apply this Helmholtz-decomposition to the two transverse components of the optical field: ${ }^{3}$

$$
\mathbf{E}_{\perp}^{\mathrm{f}}\left(\mathbf{r}_{\perp}\right)=-\nabla_{\perp} V\left(\mathbf{r}_{\perp}\right)+\left(\begin{array}{cc}
0 & 1 \\
-1 & 0
\end{array}\right) \nabla_{\perp} W\left(\mathbf{r}_{\perp}\right) .
$$

Let us now return to our equations of motion Eqs. (3)-(4). At this point it is important to notice that $\mathbf{E}^{\mathrm{f}}$ cannot be arbitrarily prescribed in all three components due to the coupling of different components via Eq. (4). Here, the Helmholtz decomposition becomes very useful, since it exactly separates fields that give rise to a longitudinal electric field component and labeled here as $\mathbf{E}^{V}$ and fields which leave the longitudinal electric field

\footnotetext{
${ }^{*}$ Any other $z=$ constant plane could also be used.
} 
component unaffected, labeled here as $\mathbf{E}^{W}$. The latter, including the labeling, becomes apparent when taking the divergence of $\mathbf{E}=\left(\mathbf{E}_{\perp}, E_{z}\right)$ and using Eq. (8):

$$
\begin{aligned}
\nabla \mathbf{E} & =\nabla_{\perp} \mathbf{E}_{\perp}+\partial_{z} E_{z} \\
& =-\Delta_{\perp} V+\partial_{z} E_{z}=0 .
\end{aligned}
$$

Here, we used the fact that $\sum_{i, j} \partial_{i} \epsilon_{i j} \partial_{j} W=0$ and thus only $V$ gives rise to the longitudinal electric field component. Note, that this decomposition corresponds to transverse electric and transverse magnetic fields. Component-wise, we can write down $\mathbf{E}_{\perp, i}^{V}=-\partial_{i} V$ and $\mathbf{E}_{\perp, i}^{W}=\sum_{j} \epsilon_{i j} \partial_{j} W$.

In summary, $\mathbf{E}_{\perp}^{V}$ is curl-free in the transverse plane and $\mathbf{E}_{\perp}^{W}$ is divergence-free in the transverse plane by construction $^{\dagger}$. The former completely determines the longitudinal electric field component and the latter does not affect it. In that sense, the two associated fields can be considered generalized radial and azimuthal polarized fields, respectively. ${ }^{4}$

Let us return to our original question [Eq. (2)], which is how can we shape $E_{z}$ with the transverse components and what is the remaining degree of freedom. Given a desired longitudinal electric field component $E_{z}$, it is clear that $\mathbf{E}_{\perp}^{V}$ is given via $\mathbf{E}_{\perp}=-\nabla_{\perp} V$ and the potential $V$ is obeys the following Poisson equation:

$$
\Delta_{\perp} V\left(\mathbf{r}_{\perp}\right)=\left.\partial_{z} E_{z}\right|_{z=0}\left(\mathbf{r}_{\perp}\right) .
$$

Because the $z$ dependence of $E_{z}$ is known [see Eq. (5)], $E_{z}^{\mathrm{f}}$ can be readily obtained in Fourier space from

$$
-\mathbf{k}_{\perp}^{2} \hat{V}\left(\mathbf{k}_{\perp}\right)=i k_{z}\left(\mathbf{k}_{\perp}\right) \hat{E}_{z}^{\mathrm{f}}\left(\mathbf{k}_{\perp}\right) .
$$

All three electric field components are fixed if we prescribe any two field components of $\mathbf{E}$ (and/or $\mathbf{B}$ ) in a plane. Thus, given that we prescribed the longitudinal electric field component $E_{z}^{f}$ only, there is exactly one degree of freedom left. This remaining degree of freedom is reflected by the fact that we can add an arbitrary (sufficiently well-behaved) field $\mathbf{E}_{\perp}^{W}$.

To fix the field $\mathbf{E}_{\perp}^{W}$, we can add an additional constraint to the field $\mathbf{E}_{\perp}$ in the transverse plane. For example, if we wanted to obtain a generalized radially polarized field (in the transverse plane), we would set $\mathbf{E}^{W}=0$. Other polarization imply similar constraints for the field $W$ : A linear transverse polarization with $\alpha E_{x}=(1-\alpha) E_{y}$ with $0 \leq \alpha \leq 1$, requires

$$
\left[\alpha k_{x}-(1-\alpha) k_{y}\right] \hat{V}\left(\mathbf{k}_{\perp}\right)=\left[\alpha k_{y}+(1-\alpha) k_{x}\right] \hat{W}\left(\mathbf{k}_{\perp}\right) ;
$$

and a circular transverse polarization, with $E_{x}= \pm i E_{y}$, requires

$$
\hat{V}\left(\mathbf{k}_{\perp}\right)= \pm i \hat{W}\left(\mathbf{k}_{\perp}\right) .
$$

In this picture, the well-known fact that any nonzero linearly or circularly polarized field necessarily gives rise to a longitudinal polarization component becomes immediately clear; in these cases $V$ is nonzero and has a nontrivial spatial dependence, and hence Eq. (10) gives rise to a nonzero $E_{z}$.

Concluding this section, we write down the full solution for given potentials $V$ and $W$ in transverse Fourier space as

$$
\hat{\mathbf{E}}\left(\mathbf{k}_{\perp}, z\right)=i\left[\left(\begin{array}{c}
-k_{x} \\
-k_{y} \\
\mathbf{k}_{\perp}^{2} \\
k_{z}\left(\mathbf{k}_{\perp}\right)
\end{array}\right) \hat{V}\left(\mathbf{k}_{\perp}\right)+\left(\begin{array}{c}
k_{y} \\
-k_{x} \\
0
\end{array}\right) \hat{W}\left(\mathbf{k}_{\perp}\right)\right] \mathrm{e}^{i k_{z}\left(\mathbf{k}_{\perp}\right) z} .
$$

\footnotetext{
${ }^{\dagger}$ The electric field in vacuum always fulfills $\nabla \cdot \mathbf{E}=0$. Hence, the restriction "in the transverse two-dimensional plane" is crucial in the present context.
} 

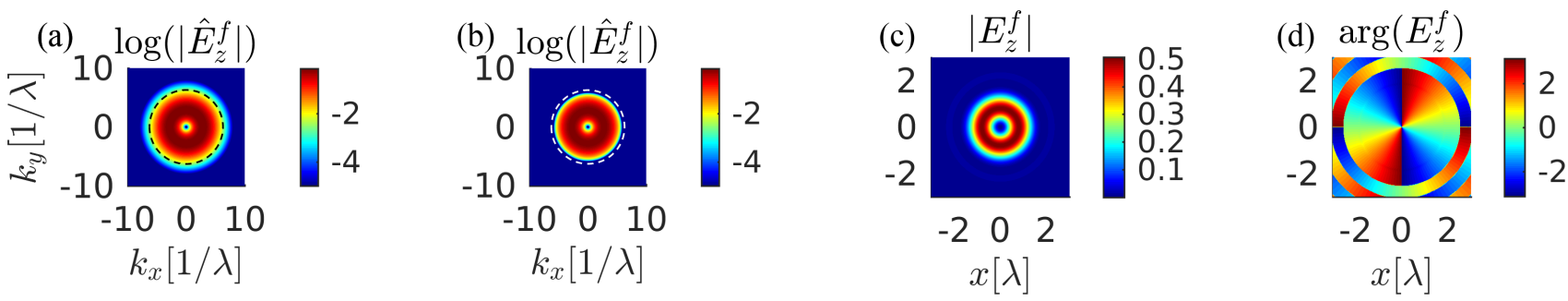

Figure 1. (a) Considering tight focusing $(\sigma=\lambda / 2)$ of Eq. (15) leads to evanescent waves as indicated by the contributions of $\left|\hat{E}_{z}^{f}\left(\mathbf{k}_{\perp}^{2} \geq k_{0}^{2}\right)\right|>0$. These can be chopped off in Fourier space (b). Filtering out components at $\mathbf{k}_{\perp}=0$ is in this case not necessary, since $E_{z}^{f}\left(\mathbf{k}_{\perp}=0\right)=0$ is already fulfilled. (c-d) show the longitudinal electric field component after filtering.

\section{ANALOGIES}

Simple analogies of the two-dimensional fields $\mathbf{E}_{\perp}$ with electro- and magnetostatics or fluid dynamics ${ }^{4}$ allow the further elucidation of the interplay between longitudinal and transverse electric field components: By considering Eq. (10) in the $z=0$-plane and associating $-\partial_{z} E_{z}(z=0)$ with a two-dimensional charge density, it is clear that we can think of the function $V$ as the respective "induced potential" and $E^{f, V}$ then emerges as the gradient field to the latter. Similarly, we can identify the potential $W$ with the only nonzero component $A_{z}^{s}$ of the magnetic vector potential, which then allows the association of the induced static magnetic field with $\mathbf{E}^{f, W}$. Another possible choice is to relate the function $W$ with the scalar stream function of fluid dynamics. These analogies are carried out in ${ }^{4}$ in detail and allow a simple intuitive approach of how one can picture the decomposition $\mathbf{E}_{\perp}^{f}=\mathbf{E}_{\perp}^{f, V}+\mathbf{E}_{\perp}^{f, W}$ and relate them to the longitudinal electric field component $E_{z}$.

\section{EXAMPLES}

This section is devoted to illustrate previous ideas with examples.

\subsection{Double charged Vortex Beam with transverse circular polarization}

As a simple first example, let us consider a double charged vortex in the longitudinal electric field component with circular transverse polarization $(V=i W)$ as follows:

$$
E_{z}^{f}\left(\mathbf{r}_{\perp}, z=0\right)=L G_{20}=\sqrt{\frac{1}{2 \pi}} \frac{(x+i y)^{2}}{\sigma^{3}} \exp \left[-\frac{r_{\perp}^{2}}{2 \sigma^{2}}\right]
$$

Let us for the following consider $\sigma=\lambda / 2$. For keeping the function Eq. (15) physical despite of decreasing the width $\sigma$ to a value that is significantly lower than the wavelength we must impose certain physical constraints: On the one hand, we must ensure that the divergence equation is satisfied for solutions propagating in the $z$-direction, which implies that $\hat{E}_{z}^{f}\left(\mathbf{k}_{\perp}=0\right)=0$ must be fulfilled. On the other hand, for finding valid bulk solutions we must impose that no evanescent fields are present, $\hat{E}_{z}^{f}\left(\mathbf{k}_{\perp}^{2} \geq k_{0}^{2}\right)=0$. In the case of Eq. (15), the first condition is satisfied automatically. For meeting the second condition we use the same filtering in spatial Fourier space as in. ${ }^{3,4}$ The effect is shown in Fig. 1(a-d).

The corresponding induced potentials $V$ and $W$ are uniquely determined, $V$ from the prescribed $E_{z}^{f}(z=0)$ and $W$ since we said in the beginning of this section that we want to consider circular polarization in the transverse electric field components, i.e. $V= \pm i W$. They are depicted in Fig. 2. Clearly, these potentials directly induce the transverse electric field components as gradient field and as field tangential to the contour lines. Given the high symmetry in the system, it is sufficient to only show the real part of $E_{x}^{f}$, since all other field components and imaginary parts are basically rotated and/or parity transformed versions of that. Figure Fig. 3 illustrates the decomposition of $\Re\left(E_{x}^{f}\right)$ into $\Re\left(E_{x}^{f, V}\right)$ and $\Re\left(E_{x}^{f, W}\right)$. Whereas the dipole $\Re\left(E_{x}^{f}\right)$ looks very simple,

\footnotetext{
${ }^{\ddagger}$ Note, that for both presented analogies are formal only, and the actual magnetic field of the vector beam differs from the one presented here.
} 

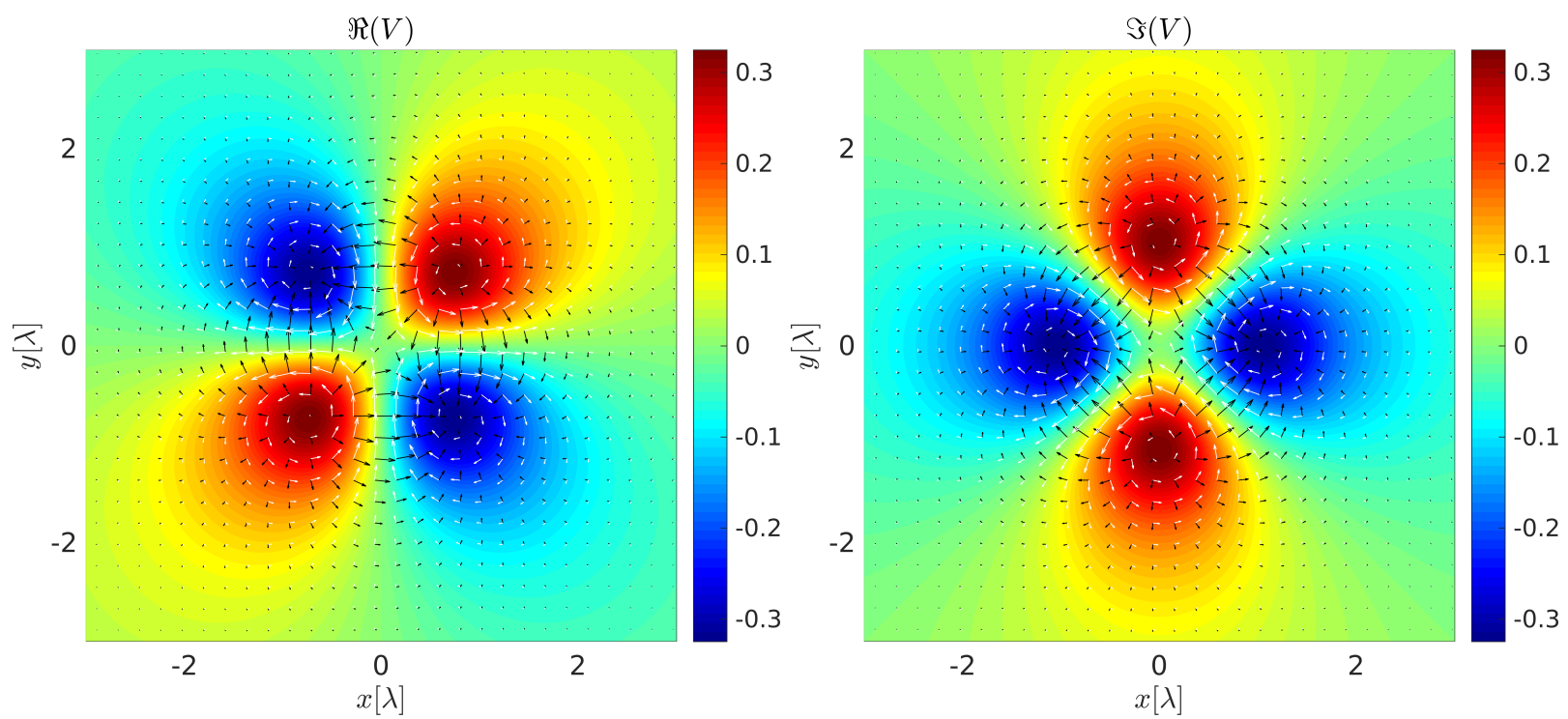

Figure 2. Induced potential $\Re(V)$ (left panel) and $\Im(V)$ (right panel) of the double charged Eq. (15). The black arrows represent the corresponding gradient field $\mathbf{E}_{\perp}^{V}=-\nabla_{\perp} V$ and the white arrows the tangential field along the contour lines $\mathbf{E}_{\perp, j}^{W} / i=-\sum_{k} \epsilon_{j k} \partial_{k} V$.
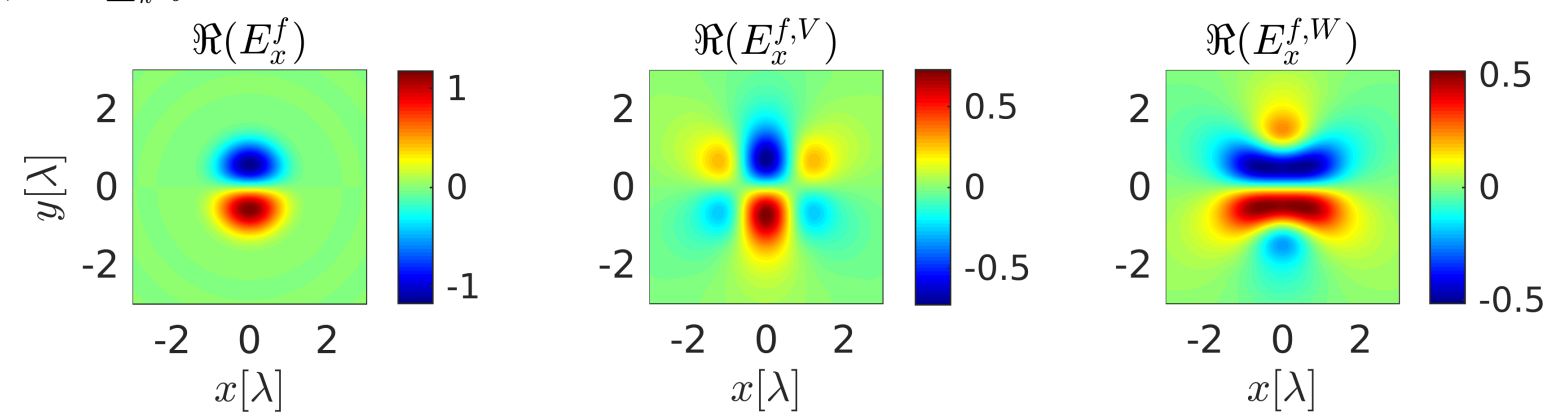

Figure 3. The real part of $E_{x}$ and its nontrivial decomposition into the real part $\Re\left(E_{x}^{f, V}\right)$ and $\Re\left(E_{x}^{f, W}\right)$ of generalized radial and azimuthal components is shown.

the corresponding generalized radial $\Re\left(E_{x}^{f, V}\right)$ and azimuthal fields $\Re\left(E_{x}^{f, W}\right)$ look a bit more involved. Note, that whereas $\Re\left(E_{x}^{f}\right)$ has roughly twice the amplitude of $\Re\left(E_{z}^{f}\right)$, one can easily reduce the amplitudes of the transverse electric field components without affecting the longitudinal electric field component by simply setting $\mathbf{E}_{\perp}^{f, W}=0$. This allows to decrease the amplitude ratio between transverse and longitudinal electric field component from roughly $2: 1$ to almost $1: 1$.

\subsection{Evanescent Trefoil}

In the previous section we considered a tightly focused double charged vortex in the longitudinal electric field component. We had to filter away evanescent waves to make the solution physical. In this section, we would like to present a thought experiment: We consider an artificial light source located in the $z=0$ plane. That allows us to consider a light distribution with a significant amount of evanescent waves in the near field. Such a distribution is physical, as the evanescent waves are damped in both $\pm z$ directions. Hence, it permits to consider the opposite case to the previous section, where we can retain the evanescent part of the beam, however we have to spectrally filter parts of the beam around the origin $k_{\perp}^{2}=0$.

Vortex knots have been studied in a range of different fields during the last two decades including water waves, ${ }^{19}$ as soliton solutions in the Skyrme-Faddev model, ${ }^{20}$ in frustrated magnets, ${ }^{21}$ in excitable media ${ }^{22-25}$ and in optics. ${ }^{3,26-28}$ A proposal for imprinting optical knots onto BECs has been made in. ${ }^{29,30}$ Propagating the light distribution shown in Fig. 4 away from the focal plane yields a vortex knot in shape of a trefoil in the 

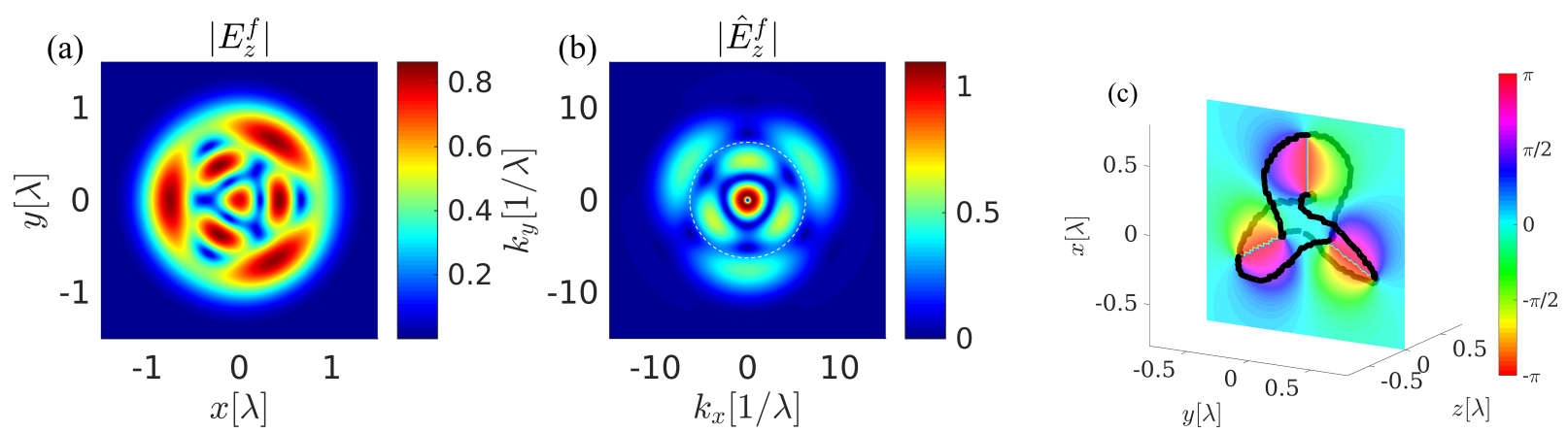

Figure 4. (a) Beam profile with high spatial complexity per voxel $\lambda^{3}$ leads to a large amount of evanescent waves (b). Whereas the thought experiment described admits these evanescent waves, we have to spectrally filter to ensure $E_{z}^{f}\left(\mathbf{k}_{\perp}=\right.$ $0)=0$, visible as the little hole in the center of (b). Propagating this function yields a vortex trefoil in the longitudinal electric field component (c).

longitudinal electric field component in the near field of the source. Note, that for the propagation evanescent waves were taken into account.

\section{CONCLUSIONS}

In this paper we have reviewed how one can realize an arbitrary (sufficiently well-behaved) longitudinal electric field component and how one can use the the remaining degree of freedom to tune the polarization in the transverse plane. We discussed evanescent waves and presented a thought experiment that allows to propagate a trefoil vortex knot in the longitudinal electric field component containing evanescent waves. Furthermore, we discussed analogies to fluid dynamics that allow to intuitively understand the relation among longitudinal and transverse electric field components.

\section{ACKNOWLEDGMENTS}

F.M, I.G.H. and S.A.G. acknowledge funding by the Leverhulme Trust Research Programme Grant RP2013-K009, F.M. acknowledges support by the Danish National Research Foundation through a Niels Bohr professorship and S.S. acknowledges support by the Qatar National Research Fund through the National Priorities Research Program (Grant No. NPRP 8-246-1-060).

\section{REFERENCES}

[1] Richards, B. and Wolf, E., "Electromagnetic diffraction in optical systems. ii. structure of the image field in an aplanatic system," Proc. Royal Soc. A 253, 358-379 (1959).

[2] Youngworth, K. S. and Brown, T. G., "Focusing of high numerical aperture cylindrical-vector beams," Opt. Express 7, 77 (2000).

[3] Maucher, F., Skupin, S., Gardiner, S. A., and Hughes, I. G., "Creating complex optical longitudinal polarization structures," Phys. Rev. Lett. 120, 163903 (2018).

[4] Maucher, F., Skupin, S., Gardiner, S. A., and Hughes, I. G., "An intuitive approach to structuring the three polarization components of light," New Journal of Physics XXX, XXX (2018).

[5] Wang, H., Shi, L., Lukyanchuk, B., Sheppard, C., and Chong, C. T., "Creation of a needle of longitudinally polarized light in vacuum using binary optics," Nature Photonics 2, 501 (2008).

[6] Qin, F., Huang, K., Wu, J., Jiao, J., Luo, X., Qiu, C., and Hong, M., "Shaping a subwavelength needle with ultra-long focal length by focusing azimuthally polarized light," Sci. Rep. 5, 9977 (2015).

[7] Freund, I., "Cones, spirals, and Möbius strips, in elliptically polarized light," Optics Communications 249(1), 7 - 22 (2005).

[8] Bauer, T., Banzer, P., Karimi, E., Orlov, S., Rubano, A., Marrucci, L., Santamato, E., Boyd, R. W., and Leuchs, G., "Observation of optical polarization Möbius strips," Science 347, 964-966 (2015). 
[9] Bauer, T., Neugebauer, M., Leuchs, G., and Banzer, P., "Optical polarization Möbius strips and points of purely transverse spin density," Phys. Rev. Lett. 117, 013601 (2016).

[10] Nieminen, T. A., Stilgoe, A. B., Heckenberg, N. R., and Rubinsztein-Dunlop, H., "Angular momentum of a strongly focused gaussian beam," Journal of Optics A: Pure and Applied Optics 10(11), 115005 (2008).

[11] Schmiegelow, C. T., Schulz, J., Kaufmann, H., Ruster, T., Poschinger, U. G., and Schmidt-Kaler, F., "Transfer of optical orbital angular momentum to a bound electron," Nat. Commun. 7, 12998 (2016).

[12] Quinteiro, G. F., Schmidt-Kaler, F., and Schmiegelow, C. T., "Twisted-light ion interaction: The role of longitudinal fields," Phys. Rev. Lett. 119, 253203 (2017).

[13] Hnatovsky, C., Shvedov, V., Krolikowski, W., and Rode, A., "Revealing local field structure of focused ultrashort pulses," Phys. Rev. Lett. 106, 123901 (2011).

[14] Rubinsztein-Dunlop et. al., H., "Roadmap on structured light," Journal of Optics 19, 013001 (2017).

[15] Andrews, D. L., ed., [Structured Light and Its Applications], Academic Press, Burlington (2008).

[16] Maucher, F., Pohl, T., Skupin, S., and Krolikowski, W., "Self-organization of light in optical media with competing nonlinearities," Phys. Rev. Lett. 116, 163902 (2016).

[17] Otte, E., Tekce, K., and Denz, C., "Tailored intensity landscapes by tight focusing of singular vector beams," Opt. Express 25, 20194-20201 (2017).

[18] Adams, C. S. and Hughes, I. G., [Optics f2f], Oxford University Press (2018).

[19] Kleckner, D. and Irvine, W. T. M., "Creation and dynamics of knotted vortices," Nature Phys. 9, 253 (2013).

[20] Battye, R. A. and Sutcliffe, P. M., "Knots as stable soliton solutions in a three-dimensional classical field theory," Phys. Rev. Lett. 81, 4798-4801 (1998).

[21] Sutcliffe, P., "Skyrmion knots in frustrated magnets," Phys. Rev. Lett. 118, 247203 (2017).

[22] Sutcliffe, P. M. and Winfree, A. T., "Stability of knots in excitable media," Phys. Rev. E 68, 016218 (2003).

[23] Maucher, F. and Sutcliffe, P., "Untangling knots via reaction-diffusion dynamics of vortex strings," Phys. Rev. Lett. 116, 178101 (2016).

[24] Maucher, F. and Sutcliffe, P., "Length of excitable knots," Phys. Rev. E 96, 012218 (2017).

[25] Binysh, J., Whitfield, C. A., and Alexander, G. P., "Stable and unstable vortex knots in excitable media," (2018).

[26] Leach, J., Dennis, M. R., Courtial, J., and Padgett, M. J., "Vortex knots in light," New Journal of Physics 7, $55(2005)$.

[27] Dennis, M. R., King, R. P., Jack, B., O'Holleran, K., and Padgett, M. J., "Isolated optical vortex knots," Nat. Phys. 6, $118-121$ (2010).

[28] Sugic, D. and Dennis, M. R., "Singular knot bundle in light," J. Opt. Soc. Am. A 35, 1987-1999 (2018).

[29] Ruostekoski, J. and Dutton, Z., "Engineering vortex rings and systems for controlled studies of vortex interactions in bose-einstein condensates," Phys. Rev. A 72, 063626 (2005).

[30] Maucher, F., Gardiner, S. A., and Hughes, I. G., "Excitation of knotted vortex lines in matter waves," New J. of Phys. 18, 063016 (2016). 